\title{
Application of Life Cycle Assessment to Optimize the MSW Classification Schemes, Pudong, China
}

\author{
Xiaojing Chen \\ School of Economics, Shanghai University, Shanghai, China \\ Email address: \\ Xiaojingchen2013@163.com \\ To cite this article: \\ Xiaojing Chen. Application of Life Cycle Assessment to Optimize the MSW Classification Schemes, Pudong, China. International Journal of \\ Environmental Monitoring and Analysis. Vol. 3, No. 5, 2015, pp. 347-350. doi: 10.11648/j.ijema.20150305.26

\begin{abstract}
In recent years, municipal solid waste management has bacame increasingly important in urban management, and waste classification is one of the very important parts in the waste management. Exactly how garbage classification and classification to what extent are the major problems in China to promote garbage classification and comprehensively promot policies faced. In this paper, we use life cycle assessment (Life Cycle Assessment, LCA) to assess the potential environmental impact of the four possible classification schemes, that source rough classification, the source of the general classification, the source detailed classification and fine classification source. Pudong New Area is in each case, and three indicators assessed: for the greenhouse effect (Global Warming, GW), acidified (Acidification) and eutrophication (Eutrophication). The results show that shame 3 is the optimal solution for the environment; followed by 2 and 4 programs; shame 1 is the worst environmental benefit program.
\end{abstract}

Keywords: Municipal Solid Waste, Waste Classification, Life Cycle Assessment

\section{基于生命周期的中国城市生活垃圾分类收集方案的评估--以中国 上海市浦东新区为例}

\section{陈肖静}

经济学院, 上海大学, 上海, 中国

\section{邮箱}

Xiaojingchen2013@163.com

摘要: 近年来, 城市生活垃圾管理在城市管理中愈发重要, 而生活垃圾分类又是垃圾管理中相当重要的一部分。究竟 将生活垃圾如何分类、分类到何种程度是中国当前促进垃圾分类全面推进政策方面所面临的主要问题。本文运用生命 周期评价法 (Life Cycle Assessment, LCA) 对四种可能的分类方案，即源头粗分类、源头一般分类、源头细分类和源 头精细分类, 在运行中对环境造成的潜在影响进行评估。每种情形均以中国上海市浦东新区为例, 并针对温室效应 (Global Warming, GW)、酸化 (Acidification) 和富营养化 (Eutrophication)三个指标进行评估。结果显示: 方案三为 环境最优方案；其次为方案二和方案四；方案一的环境效益最差。

关键词：城市生活垃圾，垃圾分类，生命周期评价法

\section{1. 引言}

美国环保署1995年称: 一个成功的综合的固体废弃物 管理体系应以实现环境效益、经济效益和社会可接受性同
时最大化为目标，包括源头减量（包括循环使用）、回收 再利用和堆肥以及最后的焚烧和填埋三大块内容。其中前 两者是最受鼓励的生活垃圾处理方式。 
近年来，随着中国经济快速发展人民生活水平不断提 高, 城市化进程也在不断加快---目前中国城镇人口占总 人口的比例已经超过 $50 \%$ 。随之而来的城市生活垃圾的产 量也在不断增加, 并成为中国经济发展的一大障碍。为了 避免 “垃圾围城” 的危机，2012年中国国务院办公厅印发 了由国家发展和改革委员会、住房和城乡建设部、环境保 护部组织编制的《“十二五” 全国城镇生活垃圾无害化处 理设施建设规划》, 这为指导各地加快生活垃圾无害化处 理设施建设和安排投资提供了重要依据。生活垃圾管理规 划很重要一个的方面是确保采取一些措施来减少特定区 域内的生活垃圾管理所造成的环境污染。而, LCA评估方 法为固体废弃物管理决策者和规划者评估生活垃圾管理 政策提供了一个极好的框架。

生命周期评价法 (LCA) 是一种评价与一个产品、工艺 或服务相关的环境因素和潜在影响的方法。它可以用来量 化一个产品或工艺 “从摇篮到坟墓” 的整个生命周期过程 对环境造成的潜在的影响。整个生命周期从原材料的开采 开始, 到产品的生产、使用直至最终分解处置为止。LCA 是一个系统性的分析工具, 在 20 世纪 90 年代该方法快速发 展, 并且实现了一定水平上的标准化与适应性。IOS 标准 和一些指导手册、准则现已生成。LCA的实现首先要汇编与 评价对象相关的能源以及原材料的投入和环境释放清单; 然后评估与清单中已识别的投入和释放相关的潜在的环境 影响; 最后通过对结果的解释来帮助决策者做出更明智的 决策。[2]

LCA方法在城市生活垃圾管理的研究中也是常用以评 估环境流量的系统的，多阶段的方法。Wei Zhao等人 [3] 应用LCA方法以中国天津市为例对现有的和可能实现的生 活垃圾管理模式关于温室气体 (GHG) 排放指标的定量评估, 结果显示对于结合了不同改进意见的综合生活垃圾管理 模式, 其GHG的释放是最高的; 并且 GHG的释放与回收率几 乎成线性负相关。R. J. Hong 等人 [4] 以中国上海市浦东新 区为例, 运用LCA评价了多种生活垃圾最终处置方案的总 的环境潜在影响, 得出未经过BMT预处理的最终处置方案 的总的环境潜在影响要比经过BMT预处理的高 1.5 倍。

Francesco Cherubini等人 [5]也运用LCA的方法探寻适合 罗马的垃圾管理方案, 在考虑全球和地方性排放、总的原 材料需求、总的能源需求以及生物足迹这四方面因素的情 况下得出填埋是最糟糕的管理方案。

\section{2. 中国上海市浦东新区生活垃圾现状}

中国上海市自 2000 年被住房和城乡建设部列为生活 垃圾分类收集试点城市以来, 对生活垃圾分类进行了探索 和尝试。2008-2-2中国上海市尝试推广以 “有害垃圾十玻 璃十可回收物十其它垃圾” 为内容的四色垃圾分类新标准; 2010年, 中国上海市发布了《关于推进生活垃圾分类促进 源头减量实施意见》、《关于进一步加强生活垃圾管理的 若干意见》等文件, 明确落实以 “大分流、小分类” 为核 心的生活垃圾分类减量体系建设, 实行家庭厨余垃圾与其 他生活垃圾的 “干湿” 分类处理; 《上海市促进生活垃圾 分类减量办法》规定，2014年5月1日起实行生活垃圾分4
类: 可回收物、有害垃圾、湿垃圾和干垃圾。可见, 为了 更好地实现生活垃圾分类, 中国上海市在不断地进行新的 尝试, 分类标准不断在变化。因此, 找到一个切实可行的 分类方案, 在浦东乃至上海都是多年来的重大课题以及急 切需求。

\section{1. 中国上海市浦东生活垃圾理化特性}

2013 年浦东新区生活垃圾总清运量为 186.8 万吨, 比 上一年增长 $7.3 \%$; 日均清运量 4822 吨/天; 人均垃圾清运 量约为 $0.96 \mathrm{~kg} /$ 人 ・ $\mathrm{d}$, 比上一年增长 $4.35 \%$ 。[6] 2013 年浦 东生活垃圾的最终处置为填埋和焚烧。根据2013年中国上 海市浦东生活垃圾理化特性, 可以得出图1。

从图1可以看出, 2013年中国上海市浦东生活垃圾清 运量中, 可回收物、厨余垃圾、竹木等混杂在一起而不能 回收再利用的混合垃圾占 $96.87 \%$, 成为主要的清运对象, 它们将直接被运送到最终处置场所进行处置。这对环境效 益造成了很大的威胁。由此可见, 垃圾分类在中国上海市 浦东新区仍是需要进一步解决的重大问题。

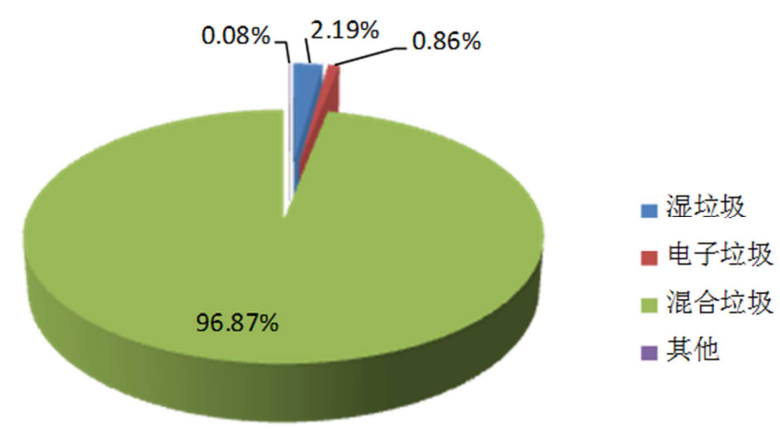

图1 2013年清运量理化特性 (统计口径为湿基干重)。

\section{2. 可选方案的提出}

借鉴日本、德国、新加坡等国外城市垃圾分类的成功 经验 [7-9], 并结合目前中国上海市浦东新区的垃圾分类 状况, 按照由粗到细的分类原则, 定义了 4 种生活垃圾分 类方案:

方案一: 源头粗分类，即将生活垃圾中的有毒有害垃 圾和厨余垃圾分出来;

方案二: 源头一般分类, 即将生活垃圾分为有毒有害 垃圾、可回收物、厨余垃圾和其他垃圾 4 类;

方案三: 源头细分类，即在方案二的基础上将可回收 物分为纸类、塑料、废金属、废玻璃等小类;

方案四: 源头精细分类, 即在方案三的基础上将每一 小类再细分类, 如将纸类再细分为报纸、书本、纸壳等。

\section{LCA评价}

\section{1. 系统性边界和假设}

在LCA中，系统边界的确定和假设的定义对最终结果 和解释部分是至关重要的。图 2 显示的是 4 种分类方案的系 统性边界。选用 1 吨的城市生活垃圾作为每种方案在生命 周期评价中的功能单位。这里运输和最终处置被认为是环 
境因素消耗和排放最多的两个环节，因此被包括在内。垃 圾桶/厢房、转运站以及在运输过程中的环境污染因无法 准确计量, 故不在边界范围内。

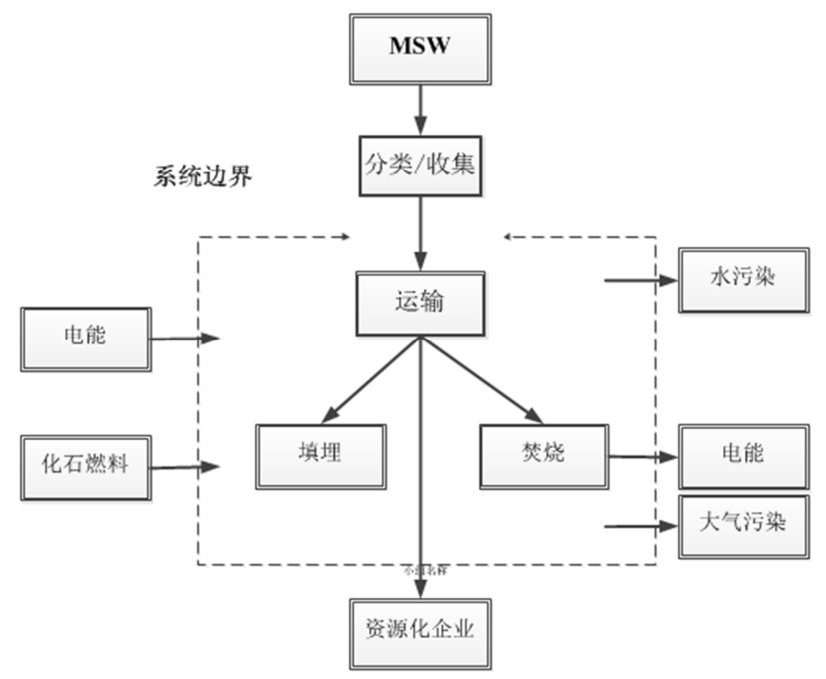

图2 垃圾分类方案系统性边界。

焚烧前需要对垃圾进行预处置以减少垃圾的体积和 水分, 这过程中会有渗滤液产生, 燃烧过程中消耗柴油, 但是在燃烧过程中能产生电能, 废气是经过空气净化装置 后排出的, 主要有酸性气体、二噁英和飞灰等; 填埋过程 中会有渗滤液和大量废气产生, 主要有甲烷, 大约占总量 的 50-70\%; 其次为碳氧化物, 约占到 $30-50 \%$; 还有少部分 的 $\mathrm{NH}_{3} 、 \mathrm{H}_{2} \mathrm{~S}$ 和其他无机气体。

\section{2. 生命周期清单}

表1显示了中国上海市浦东生活垃圾转运和最终处置 生命周期清单。能源消耗、废气排放 $\left(\mathrm{CO}_{2}, \mathrm{CH}_{4}, \mathrm{CO}, \mathrm{NOx}, \mathrm{SO}_{2}, \mathrm{HCL}, \mathrm{HF}, \mathrm{H}_{2} \mathrm{~S}, \mathrm{NH}_{3}\right.$ 等) 和水污染 (P, N) 均被评估。交通运输排放与运行时的加速、减速和所使用 燃料密切相关。[10]这里以 $15 \mathrm{t}$ 厢式柴油垃圾车为例, 平 均运距为 87.6 公里计算。人类活动的 $\mathrm{CO}_{2}$ 排放没有计算在 内, 但是化石燃料的燃烧排放的 $\mathrm{CO}_{2}$ 包括在内。

表1 生活垃圾转运和最终处置生命周期清单 (1t)。

\begin{tabular}{lll|l}
\hline & 填埋 $[4]$ & 焚烧 [4] & 运输 [10] \\
\hline 能源 $(\mathrm{MJ} / \mathrm{t})$ & $8.03 \times 10$ & $-3.17 \times 10^{2}$ & 2.83 \\
$\mathrm{C} 02(\mathrm{~kg} / \mathrm{t})$ & $9.04 \times 10$ & $5.0 \times 10^{2}$ & -- \\
$\mathrm{CH} 4(\mathrm{~kg} / \mathrm{t})$ & $1.82 \times 10$ & -- & -- \\
$\mathrm{C} 0(\mathrm{~kg} / \mathrm{t})$ & $1.3 \times 10^{-3}$ & $5.7 \times 10^{-2}$ & $2.9 \times 10^{-2}$ \\
$\mathrm{NOx}(\mathrm{kg} / \mathrm{t})$ & -- & $2.5 \times 10^{-1}$ & $3.8 \times 10^{-2}$ \\
$\mathrm{~S} 02(\mathrm{~kg} / \mathrm{t})$ & $1.0 \times 10^{-4}$ & $3.3 \times 10^{-1}$ & -- \\
$\mathrm{Hc} 1(\mathrm{~kg} / \mathrm{t})$ & $6.7 \times 10^{-3}$ & $7.0 \times 10^{-3}$ & -- \\
$\mathrm{HF}(\mathrm{kg} / \mathrm{t})$ & $1.3 \times 10^{-3}$ & $1.0 \times 10^{-3}$ & -- \\
$\mathrm{H} 2 \mathrm{~S}(\mathrm{~kg} / \mathrm{t})$ & $3.7 \times 10^{-2}$ & -- & -- \\
$\mathrm{NH} 3(\mathrm{~kg} / \mathrm{t})$ & $3.4 \times 10^{-2}$ & -- & -- \\
$\mathrm{N}(\mathrm{kg} / \mathrm{t})$ & $3.9 \times 10^{-2}$ & $3.0 \times 10^{-4}$ & -- \\
$\mathrm{P}(\mathrm{kg} / \mathrm{t})$ & $2.0 \times 10^{-3}$ & $2.9 \times 10^{-5}$ & -- \\
$\mathrm{THC}(\mathrm{kg} / \mathrm{t})$ & -- & -- & $1.1 \times 10^{-2}$ \\
\hline
\end{tabular}

由于分类方案设计的分类条目详细程度不同, 故所需 转运所要使用的车辆数目也不一样。混合收运时不用区分 类别, 故运送效率最高。根据以往的运输经验, 假设方案 一、方案二、方案三和方案四的运输所需分别为混合运输 时的 $1.05 ; 1.1 ; 1.2$ 和 1.5 倍, 最终处置以焚烧和填埋各 0.5 为权重处理。结合R. J. Hong等人 [4]对最终处置的清 单统计和Changhong Chen等人 [10]对重型柴油卡车排放 的数据统计, 再加上每种方案的减量贡献, 可以得出每种 方案每一单位生活垃圾的LCA清单, 如表2所示。

表2 4 种方案的LCA清单 (1t)。

\begin{tabular}{|c|c|c|c|c|}
\hline & 方案一 & 方案二 & 方案三 & 方案四 \\
\hline 能源 $(M J / t)$ & $-1.10 \times 10^{2}$ & $-1.08 \times 10^{2}$ & $-1.07 \times 10^{2}$ & $-1.06 \times 10^{2}$ \\
\hline $\mathrm{CO}_{2}(\mathrm{~kg} / \mathrm{t})$ & $2.82 \times 10^{2}$ & 2. $77 \times 10^{2}$ & 2. $76 \times 10^{2}$ & $2.75 \times 10^{2}$ \\
\hline $\mathrm{CH}_{4}(\mathrm{~kg} / \mathrm{t})$ & 8.68 & 8.53 & 8.51 & 8.46 \\
\hline $\mathrm{CO}(\mathrm{kg} / \mathrm{t})$ & $5.82 \times 10^{-2}$ & $5.92 \times 10^{-2}$ & $6.20 \times 10^{-2}$ & $7.06 \times 10^{-2}$ \\
\hline $\mathrm{NO}_{\mathrm{x}}(\mathrm{kg} / \mathrm{t})$ & 1. $59 \times 10^{-1}$ & 1. $59 \times 10^{-1}$ & 1. $63 \times 10^{-1}$ & 1. $74 \times 10^{-1}$ \\
\hline $\mathrm{SO}_{2}(\mathrm{~kg} / \mathrm{t})$ & 1. $57 \times 10^{-1}$ & $1.55 \times 10^{-1}$ & 1. $54 \times 10^{-1}$ & 1. $54 \times 10^{-1}$ \\
\hline $\operatorname{HCL}(\mathrm{kg} / \mathrm{t})$ & $6.53 \times 10^{-3}$ & $6.42 \times 10^{-3}$ & $6.40 \times 10^{-3}$ & $6.37 \times 10^{-3}$ \\
\hline $\mathrm{HF}(\mathrm{kg} / \mathrm{t})$ & 1. $10 \times 10^{-3}$ & 1. $08 \times 10^{-3}$ & 1. $07 \times 10^{-3}$ & 1. $07 \times 10^{-3}$ \\
\hline $\mathrm{H}_{2} \mathrm{~S}(\mathrm{~kg} / \mathrm{t})$ & 1. $76 \times 10^{-2}$ & 1. $74 \times 10^{-2}$ & 1. $73 \times 10^{-2}$ & 1. $72 \times 10^{-2}$ \\
\hline $\mathrm{NH}_{3}(\mathrm{~kg} / \mathrm{t})$ & 1. $62 \times 10^{-2}$ & $1.59 \times 10^{-2}$ & 1. $59 \times 10^{-2}$ & 1. $58 \times 10^{-2}$ \\
\hline $\mathrm{N}(\mathrm{kg} / \mathrm{t})$ & 1. $87 \times 10^{-2}$ & $1.84 \times 10^{-2}$ & 1. $84 \times 10^{-2}$ & $1.83 \times 10^{-2}$ \\
\hline$P(\mathrm{~kg} / \mathrm{t})$ & $9.68 \times 10^{-4}$ & $9.51 \times 10^{-4}$ & $9.48 \times 10^{-4}$ & 9. $44 \times 10^{-4}$ \\
\hline $\mathrm{THC}(\mathrm{kg} / \mathrm{t})$ & 1. $15 \times 10^{-2}$ & $1.21 \times 10^{-2}$ & 1. $32 \times 10^{-2}$ & 1. $65 \times 10^{-2}$ \\
\hline
\end{tabular}

\section{4. 生命周期影响分析}

城市生活垃圾管理中要涉及到许多的环境因素。这里 我们只考虑了能源消耗、废气排放和水污染, 并且将这些 污染因子量化为酸化 (AP) 、温室效应 (GWP) 和富营养化 (NE) 这三大潜在的环境污染类别。标准化参数和权重因子 均根据EDIP方法得到。参考Mendes et al., 2004选取当量 因子。每种方案的能源消耗和排放均被量化为GWP、NE和 $\mathrm{AP}$ 三大特征类别。

图3显示了每种分类方案下 $1 t$ 生活垃圾所造成的潜 在的温室效应影响（ $\mathrm{kg} \mathrm{CO} 2$ equiv/t MSW）。方案一下 的 $1 \mathrm{t}$ 生活垃圾的 GWP指标最高, 约为 $498 \mathrm{~kg} \mathrm{CO} 2$ equiv / t MSW。因为方案一情形只对生活垃圾进行源头粗分类, 因此回收再利用率比较低, 减量贡献率也很低。这就使 得生活垃圾绝大多数都被运往焚烧厂和填埋场进行最终 处置, 垃圾焚烧会产生大量的C排放, 填埋也会有碳氧化 物的排放, 而车辆运输的 $C$ 排放相比来说较小, 因此方案 一的GWP值很高。随着分类方案的细化, 垃圾的资源化程 度不断提高, 相应的GWP值也会逐渐降低。因此方案四的 GWP值是最低的, 约为 $486 \mathrm{~kg} \mathrm{CO}$ equiv/tMSW, 比方案 一降低了约 $2 \%$ 。

图4显示了每种方案下 $1 \mathrm{t}$ 生活垃圾所造成的潜在的酸 化影响（ $\mathrm{kg} \mathrm{\textrm {SO } _ { 2 }}$ equiv/t MSW）。又方案一到方案四, $\mathrm{AP}$ 值呈现了先下降后上升的趋势。这是因为酸化影响因素 主要来源于焚烧和转运过程中的酸性气体排放。方案一到 方案二AP值呈下降趋势是因为回收率增大, 焚烧产生的酸 性气体减少量大于转运过程排放的增加量。由方案二到方 案四, 尽管回收率也在不断增大, 但是转运过程排放的酸 性气体增加量大于焚烧产生的酸性气体减少量, 因此AP 
呈上升趋势, 甚至方案四的AP值要大于方案一, 成为最高 值 (约为 $0.344 \mathrm{~kg} \mathrm{SO}$ equiv/tMSW)。

图5显示了每种方案下 $1 \mathrm{t}$ 生活垃圾所造成的潜在的富 营养化影响（ $\mathrm{kg} \mathrm{NO} \mathrm{N}_{3}-$ equiv / t MSW）。与图4的走势非 常相似, 先下降后上升。这主要是由于转运过程中会排放 $\mathrm{NO}_{x}$, 而 $\mathrm{NO}_{x}$ 是影响富营养化的主要因素。方案四又为最高 值0.403。

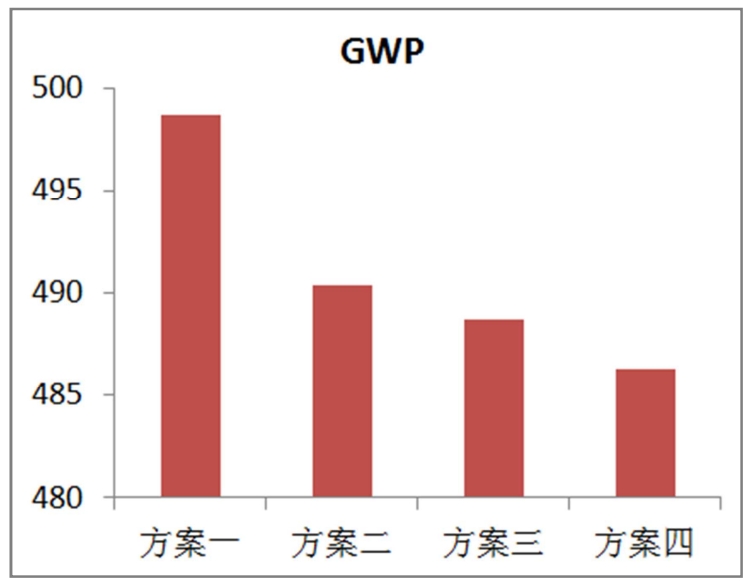

图3 1t生活垃圾在每种方案下潜在的GWP。

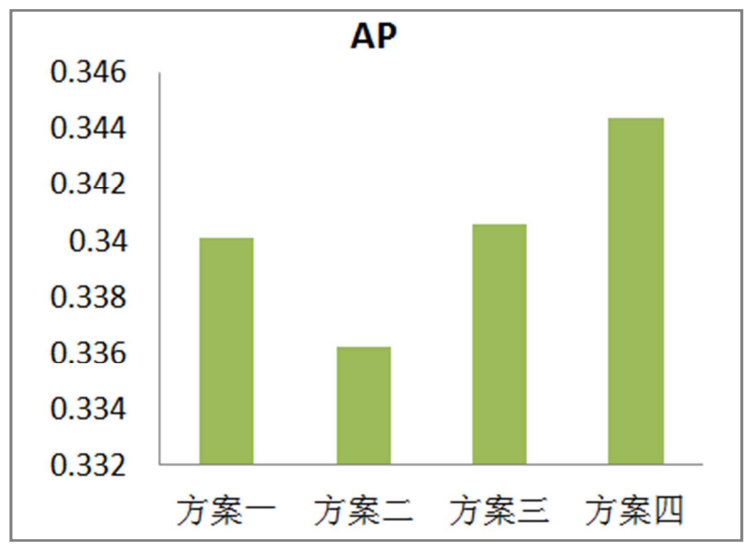

图4 1t生活垃圾在每种方案下潜在的AP。

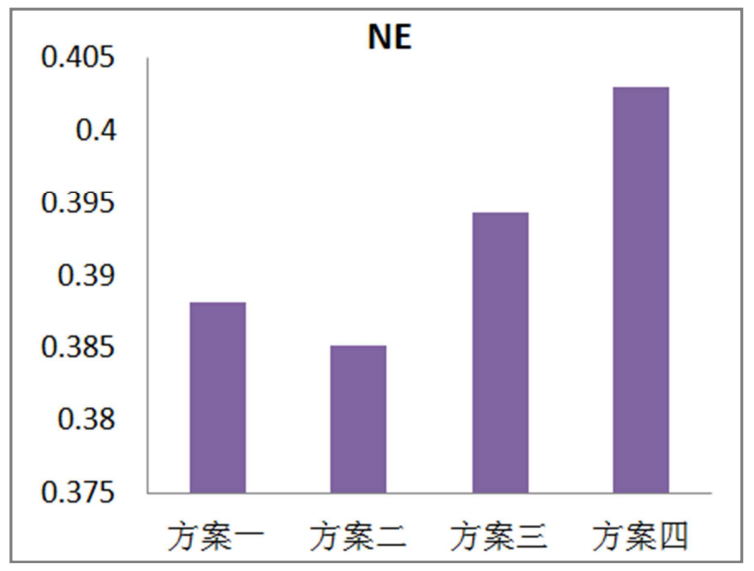

图5 1t生活垃圾在每种方案下潜在的NE。

\section{5. 结论与分析}

4 种分类方案的生命周期评价结果显示, 方案一的GWP 值是最高的; 方案二的AP值和NE值是最小的; 方案的GWP 值是最小的, 而AP和NE值是最高的。方案三的TEIP值最小 (0.01879); 其次为方案二, 为 0.01880 ; 然后是与方案 二很接近的方案四, 为 0.01881 ; 方案一最高 (0.01909)。 由此可见, 方案三应为中国上海市浦东新区生活垃圾 4 种 分类方案中环境潜在影响最低的方案, 也即环境效益最高 方案。

\section{参考文献}

[1] 蒋玉章. 中国与发达国家垃圾分类开展情况分析 [J]. 资源 与环境, 2012.10。

[2] Life Cycle Assessment: Principles and Practice by Scientific Applications International Corporation (SAIC). US-EPA, Contract N0. 68-C02-067, 2006 May.

[3] Wei Zhao, Ester van der Voet, Yu Zhang, et al., Life cycle assessment of municipal solid waste management with regard to greenhouse gas emissions: Case study of Tianjin, China. Science of the Total Environment, 2009, 407:1517-1526.

[4] R. J. Hong, G. F. Wang, R. Z. Guo, et al. . Life cycle assessment of BMT-based integrated municipal solid waste management: Case study in Pudong, China. Resources, Conservation and Recycling, 2006, 49:129-146.

[5] Francesco Cherubini, Silvia Bargigli, Sergio Ulgiati. Life cycle assessment of waste management strategies: Landfilling, sorting plant and incineration. Energy, 2009, 34:2116-2123.

［6］2013浦东新区废弃物管理年度报告。

[7] 王子彦, 丁旭, 周丹. 中国城市生活垃圾分类回收问题研究 一对日本城市垃圾分类经验的借鉴 $[J]$. 东北大学学 报, 2008, 10:501-504。

［8］贺俏毅, 陈松. 加拿大城市生活垃圾分类经验对中国的启示 [M]. 城市规划和科学发展. 2009.9。

[9] 唐艳冬, 杨玉川, 王树堂等. 借鉴国际经验推进中国农村生 活垃圾管理 $[J]$. 环境保护，2014，42（14）：70-73。

[10] Changhong Chen, Cheng Huang, Qiguo Jing, et al.. On-road emission characteristics of heavy-duty diesel vehicles in Shanghai. Atmospheric Environment, 2007, 41:5334-5344. 